\title{
Condições Necessárias para Problemas de Otimização com Tempo Contínuo sob Regularidade Tipo Independência Linear
}

Moisés Rodrigues Cirilo do Monte ${ }^{1}$

Doutorando em Matemática, UNESP - Câmpus S. J. Rio Preto, SP

Valeriano Antunes de Oliveira ${ }^{2}$

Departamento de Matemática Aplicada, UNESP, Câmpus S. J. Rio Preto, SP

Resumo. Condições necessárias de otimalidade de primeira e segunda ordem são obtidas para problemas de programação não-linear com tempo contínuo com restrições de igualdade e desigualdade, mediante uma qualificação de restrições tipo Independência Linear e o uso de um Teorema da Função Implícita Uniforme.

Palavras-chave. Programação Não-Linear, Tempo Contínuo, Condições Necessárias de Otimalidade, Qualificações de Restrições.

\section{Introdução}

Considere o problema de programação não-linear com tempo contínuo com restrições de igualdade e desigualdade

$$
\begin{array}{ll}
\text { Minimizar } & P(z)=\int_{0}^{T} \phi(z(t), t) d t \\
\text { sujeito a } & h(z(t), t)=0 \text { q.t.p. em }[0, T], \\
& g(z(t), t) \geq 0 \text { q.t.p. em }[0, T],
\end{array}
$$

onde $h: \mathbb{R}^{n} \times[0, T] \rightarrow \mathbb{R}^{p}, g: \mathbb{R}^{n} \times[0, T] \rightarrow \mathbb{R}^{m}, z$ é função vetorial $n$ dimensional com cada componente limitada, Lebesgue mensurável em $[0, T], T$ finito, $z(t)$ é um vetor coluna em $\mathbb{R}^{n}$ para cada $t \in[0, T]$ e usaremos ${ }^{\prime}$ para denotar vetores e matrizes transpostas. A norma em $L_{n}^{\infty}[0, T]$ é dada por

$$
\|z\|_{n}^{\infty}=\max _{1 \leq k \leq n}\left\|z_{k}\right\|^{\infty}=\max _{1 \leq k \leq n} \operatorname{ess} \sup _{t \in[0, T]}\left|z_{k}(t)\right| .
$$

A norma em $L_{m}^{1}[0, T]$ é dada por

$$
\|z\|_{m}^{1}=\max _{1 \leq k \leq m}\left\|z_{k}\right\|^{1}=\max _{1 \leq k \leq m} \int_{0}^{T}\left|z_{k}(t)\right| d t .
$$

\footnotetext{
${ }^{1}$ moises.monte@ufu.br

22antunes@ibilce.unesp.br
} 
Problemas de otimização com tempo contínuo foram inicialmente propostos por Bellman [1] em suas investigações, em programação linear com tempo contínuo, de alguns modelos dinâmicos de produção e inventário chamados "Problema de Gargalo". Uma das aplicações apresentadas nos trabalhos de Bellman é o de determinar a política de alocação que maximiza a quantidade de aço no estoque de uma indústria, ao final de um certo período.

Na literatura, os problemas com tempo contínuo são formulados apenas com restrições de desigualdade, na maioria dos casos. Mas há exceções, como por exemplo, Zalmai [6], onde condições suficientes são obtidas para problemas com restrições de igualdade e desigualdade, sob hipóteses de pseudoconvexidade estrita sobre as restrições.

Em Reiland [4], um problema com tempo contínuo com restrições de desigualdade é apresentado e condições necessárias tipo Karush-Kuhn-Tucker são obtidas mediante a utilização de um Teorema de Farkas Generalizado [2] e de uma qualificação de restrições geométrica.

Neste trabalho, propomos o uso de uma qualificação de restrição tipo Independência Linear e de um Teorema da Função Implícita Uniforme [3], para obtenção de condições necessárias de primeira e segunda ordens para o problema (PTC).

\section{Preliminares}

Denotaremos o conjunto factível por

$$
\Omega=\left\{z \in L_{n}^{\infty}[0, T] \mid h(z(t), t)=0 \text { e } g(z(t), t) \geq 0 \text { q.t.p. em }[0, T]\right\} .
$$

Definição 2.1. Dizemos que $\bar{z} \in \Omega$ é uma solução ótima local para o problema $(P T C)$ se existe $\epsilon>0$ tal que $P(\bar{z}) \geq P(z)$ para todo $z$ que satisfaça $z(t) \in \bar{z}(t)+\epsilon \bar{B}$ q.t.p. em $[0, T]$, onde $\bar{B}$ denota a bola unitária fechada.

Seja $\bar{z}$ uma solução ótima local para (PTC) e consideremos válidas as seguintes hipóteses:

(H1) $\phi(\cdot, t)$ é uma função escalar continuamente diferenciável ao longo de $[0, T], \phi(z, \cdot)$ é mensurável para cada $z$ e existe $K_{\phi}>0$ tal que

$$
\|\nabla \phi(\bar{z}(t), t)\| \leq K_{\phi} \text { q.t.p. em }[0, T] .
$$

(H2) $h(z, \cdot)$ e $g(z, \cdot)$ são mensuráveis para cada $z, h(\cdot, t)$ e $g(\cdot, t)$ são duas vezes continuamente diferenciáveis em $\bar{z}(t)+\epsilon \bar{B}$ q.t.p. em $[0, T]$.

(H3) Existe uma função crescente $\bar{\theta}:(0, \infty) \rightarrow(0, \infty), \bar{\theta}(s) \downarrow 0$ quando $s \downarrow 0$, tal que para todo $\tilde{z}, z \in \bar{z}(t)+\epsilon \bar{B}$ e q.t.p. em $[0, T]$,

$$
\|\nabla[h, g](\tilde{z}, t)-\nabla[h, g](z, t)\| \leq \bar{\theta}(\|\tilde{z}-z\|) .
$$

Existe $K_{0}>0$ tal que para q.t.p. em $[0, T]$,

$$
\|\nabla[h, g](\bar{z}, t)\| \leq K_{0} .
$$


(H4) Existe $K>0$ tal que, para q.t.p. em $[0, T]$,

$$
\operatorname{det}\left\{\Upsilon(t) \Upsilon^{\prime}(t)\right\} \geq K
$$

onde

$$
\Upsilon(t)=\left(\begin{array}{cc}
\nabla h(\bar{z}(t), t) & 0 \\
\nabla g(\bar{z}(t), t) & \operatorname{diag}\left\{-2 \sqrt{g_{i}(\bar{z}(t), t)}\right\}_{i \in\{1, \ldots, m\}}
\end{array}\right) .
$$

As hipóteses (H2)-(H4) são fundamentais na utilização do Teorema da Função Implícita Uniforme [3]. A hipótese (H4) é uma versão uniforme da qualificação de restrições independência linear da otimização não-linear em dimensão finita.

\section{Problema Irrestrito e com Restrições de Igualdade}

Considere o problema (PTC), com $h$ e $g$ identicamente nulas, isto é,

$$
\begin{array}{ll}
\text { Minimizar } & P(z)=\int_{0}^{T} \phi(z(t), t) d t \\
\text { sujeito a } & z \in \Omega,
\end{array}
$$

onde $\Omega=L_{n}^{\infty}[0, T]$. Como o problema é irrestrito, apenas utilizaremos a hipótese (H1).

Teorema 3.1 ([4], Teorema 2). Se

$$
\delta P(z ; \gamma)=\int_{0}^{T} \nabla \phi^{\prime}(z(t), t) \gamma(t) d t>0
$$

onde $z, \gamma \in L_{n}^{\infty}[0, T]$, então existe um escalar positivo $\sigma$ tal que

$$
P(z+\tau \gamma)>P(z), \text { para } 0<\tau \leq \sigma .
$$

Proposição 3.1. Se ż é uma solução ótima local para $(S R)$ então

$$
\nabla \phi(\bar{z}(t), t)=0 \text { q.t.p. em }[0, T] .
$$

Se $\phi$ for duas vezes continuamente diferenciável ao longo de [0,T], então

$$
\int_{0}^{T} \gamma^{\prime}(t) \nabla^{2} \phi(\bar{z}(t), t) \gamma(t) d t \leq 0 \quad \forall \gamma \in L_{n}^{\infty}[0, T] .
$$

Demonstração. Faz-se uso do Teorema 3.1 e de ferramentas de Análise Funcional.

Agora, considere o problema (PTC), com $g$ identicamente nula, ou seja,

$$
\begin{array}{ll}
\text { Minimizar } & P(z)=\int_{0}^{T} \phi(z(t), t) d t \\
\text { sujeito a } & h(z(t), t)=0 \text { q.t.p. em }[0, T],
\end{array}
$$

onde $\Omega=\left\{z \in L_{n}^{\infty}[0, T] \mid h(z(t), t)=0\right.$ q.t.p. em $\left.[0, T]\right\}$. Note que, como $g \equiv 0$ em (RI), as hipóteses (H2)-(H4) incidem apenas sobre $h$ e temos que $\Upsilon(t)=\nabla h(z(t), t)$. 
Teorema 3.2. Seja $\bar{z}$ minimizador local de (RI) e suponha que as hipóteses (H1)-(H4) são satisfeitas. Então, existe um único $u \in L_{p}^{\infty}[0, T]$ tal que

$$
\nabla \phi(\bar{z}(t), t)+\nabla h^{\prime}(\bar{z}(t), t) u(t)=0 \text { q.t.p. em }[0, T] .
$$

Além disso, existe $M>0$ tal que

$$
\|u(t)\| \leq M K_{0} K_{\phi} \text { q.t.p. em }[0, T] .
$$

Se $\phi(\cdot, t)$ e $h(\cdot, t)$ forem duas vezes contiuamente diferenciáveis ao longo de $[0, T]$, então

$$
\int_{0}^{T} \gamma^{\prime}(t)\left\{\nabla^{2} \phi(z(t), t)+\nabla^{2} h^{\prime}(z(t), t) u(t)\right\} \gamma(t) d t \leq 0 \quad \forall \gamma \in N
$$

onde $N$ é dado por

$$
N=\left\{\gamma \in L_{n}^{\infty}[0, T] \mid \nabla h(\bar{z}(t), t) \gamma(t)=0 \text { q.t.p. em }[0, T]\right\} .
$$

Demonstração. Sejam $\epsilon>0$ dado pelas hipóteses e $\bar{z}$ minimizador local para o problema (RI) em $\bar{z}(t)+\epsilon \bar{B}$ q.t.p. em $[0, T]$. A demonstração será feita em vários passos.

PASSO 1: Vamos definir uma aplicação que satisfaça as condições do Teorema da Função Implícita Uniforme [3]. Escolha $S_{0} \subset[0, T]$ o maior subconjunto tal que cada uma das condições em $(H 1)-(H 4)$ não se verificam, para todo $t \in S_{0}$. Por hipótese, $S_{0}$ tem medida nula. Segue de ( [5], p. 309) que existe um conjunto de Borel $S$, que é a intersecção de uma coleção enumerável de conjuntos abertos, tal que $S_{0} \subset S$ e $S \backslash S_{0}$ tem medida nula. Assim, $S$ é um conjunto de Borel de medida nula. Seja $A=[0, T] \backslash S$ um conjunto de Borel de medida total.

Defina $\mu: \mathbb{R}^{n} \times \mathbb{R}^{p} \times[0, T] \rightarrow \mathbb{R}^{p}$ dada por

$$
\mu(\xi, \eta, t)=h\left(\bar{z}(t)+\xi+\Upsilon^{\prime}(t) \eta, t\right) .
$$

Note que, para q.t.p. em $[0, T]$ e $(\xi, \eta)=(0,0), \mu(0,0, t)=h(\bar{z}(t), t)=0$ e considerando $\alpha=\min \left\{\frac{\epsilon}{2}, \frac{\epsilon}{2 K_{0}}\right\}$,

$$
\left\|\bar{z}(t)+\xi+\Upsilon^{\prime}(t) \eta-\bar{z}(t)\right\|=\left\|\xi+\Upsilon^{\prime}(t) \eta\right\| \leq\|\xi\|+\left\|\Upsilon^{\prime}(t)\right\| \cdot\|\eta\|<\epsilon,
$$

sempre que $(\xi, \eta) \in(0,0)+\alpha \bar{B}$. Isso implica que $\mu$ é diferenciável em $(0,0)+\alpha B, \forall t \in A$. Usando (H1)-(H4), temos que $\mu$ satisfaz as hipóteses do Teorema da Função Implicita Uniforme. Em particular, note que

$$
\nabla_{\eta} \mu(0,0, t)=\nabla h(\bar{z}(t), t) \Upsilon^{\prime}(t)=\Upsilon(t) \Upsilon^{\prime}(t) .
$$

Assim, pela hipótese $(\mathrm{H} 4), \nabla_{\eta} \mu(0,0, t)$ é não singular para cada $t \in A$ e junto com a hipótese (H3) resulta que existe $M>0$ tal que

$$
\left\|\left[\Upsilon(t) \Upsilon^{\prime}(t)\right]^{-1}\right\| \leq M \text { q.t.p. em }[0, T] .
$$


Então, o Teorema da Função Implícita Uniforme nos garante que existem $\sigma \in(0, \epsilon)$, $\delta \in(0, \epsilon)$ e uma aplicação implícita

$$
d: \sigma B \times A \rightarrow \delta B
$$

tal que $d(\xi, \cdot)$ é uma função mensurável para fixo $\xi$, as funções $\{d(\cdot, t) \mid t \in A\}$ são contínuas Lipschitz com constante Lipschitz comum, $d(\cdot, t)$ é continuamente diferenciável para cada $t \in A$,

$$
\begin{gathered}
d(0, t)=0 \text { q.t.p. em }[0, T], \\
\mu(\xi, d(\xi, t), t)=0 \text { q.t.p. em }[0, T], \xi \in \sigma B \\
\nabla d(0, t)=-\left[\Upsilon(t) \Upsilon^{\prime}(t)\right]^{-1} \nabla h(\bar{z}(t), t) .
\end{gathered}
$$

Escolha $\sigma_{1}>0$ e $\delta_{1}>0$ tais que

$$
\sigma_{1} \in\left(0, \min \left\{\sigma, \frac{\epsilon}{2}\right\}\right), \quad \delta_{1} \in\left(0, \min \left\{\delta, \frac{\epsilon}{2}\right\}\right), \quad \sigma_{1}+K_{0} \delta_{1} \in\left(0, \frac{\epsilon}{2}\right),
$$

onde $K_{0}$ é dado em (H3). Nos passos seguintes e sem perda de generalidade, consideraremos a função implícita $d$ definida em $\sigma_{1} B \times[0, T]$ tomando valores em $\delta_{1} B$.

Passo 2: Pode-se mostrar que, se $\bar{z}$ é um minimizador local para (RI), então $\bar{z}$ é minimizador local para o problema auxiliar dado por

$$
\begin{array}{ll}
\text { Maximizar } & \tilde{P}(z)=\int_{0}^{T} \varphi(z(t), t) d t \\
\text { sujeito a } & z \in \Omega,
\end{array}
$$

onde $\Omega=L_{n}^{\infty}[0, T]$ e $\varphi(z(t), t)=\phi\left(z(t)+\Upsilon^{\prime}(t) d(z(t)-\bar{z}(t), t), t\right)$.

Passo 3: Vamos aplicar a Proposição 3.1 para o problema (AUX). Sendo $\bar{z}$ minimizador local para (RI), então $\bar{z}$ é um minimizador local para (AUX) pelo Passo 2. Então, pela Proposição 3.1 temos que, para quase todo $t \in[0, T]$,

$$
\nabla \varphi(\bar{z}(t), t)=0 \Leftrightarrow \nabla \phi(\bar{z}(t), t)+\nabla h^{\prime}(\bar{z}(t), t) u(t)=0
$$

onde

$$
u(t)=-\left[\Upsilon(t) \Upsilon^{\prime}(t)\right]^{-1} \Upsilon(t) \nabla \phi(\bar{z}(t), t) \text { q.t.p. em }[0, T] .
$$

Note que $u \in L_{p}^{\infty}[0, T]$ é único e

$$
\|u(t)\| \leq M K_{0} K_{\phi} \text { q.t.p. em }[0, T]
$$

segue das hipóteses (H1), (H3) e de (3). Por fim, se $\phi(\cdot, t)$ e $h(\cdot, t)$ forem duas vezes contiuamente diferenciáveis ao longo de $[0, T]$, então as funções $\mu$ e $d$ são também duas vezes continuamente diferenciáveis ao longo de $[0, T]$ e, usando (5), temos para q.t.p. em $[0, T]$ e $\xi \in \sigma B$, que

$$
\nabla^{2} d(0, t)=-\left[\Upsilon(t) \Upsilon^{\prime}(t)\right]^{-1} \nabla^{2} h(\bar{z}(t), t)\left\{I_{n}-\Upsilon^{\prime}(t)\left[\Upsilon(t) \Upsilon^{\prime}(t)\right]^{-1} \Upsilon(t)\right\}
$$


Agora, pela Proposição 3.1, temos que

$$
\int_{0}^{T} \gamma^{\prime}(t) \nabla^{2} \varphi(z(t), t) \gamma(t) d t \leq 0 \quad \forall \gamma \in L_{n}^{\infty}[0, T] .
$$

Em particular, vale para $\gamma \in N$. Derivando $\varphi$ duas vezes, usando (8), o fato de que $\gamma \in N$, fazendo o produto a esquerda por $\gamma^{\prime}(t)$ e à direita por $\gamma(t)$ para q.t.p. em $[0, T]$ e integrando de 0 a $T$, obtemos (2).

\section{Problema com Restrições de Igualdade e Desigualdade}

Defina, para cada $t \in[0, T]$, o conjunto de índices de restrições de desigualdade ativas em $\bar{z}$ por

$$
I_{a}(t)=\left\{j \in\{1, \ldots, m\} \mid g_{j}(\bar{z}(t), t)=0\right\} .
$$

Teorema 4.1. Seja $\bar{z}$ minimizador local para o problema (PTC). Suponha que as hipóteses (H1)-(H4) são satisfeitas e que $g(\bar{z}(\cdot), \cdot)$ é limitada em $[0, T]$. Então, existem $u \in L_{p}^{\infty}[0, T]$ e $v \in L_{m}^{\infty}[0, T], v_{i}(t) \geq 0$ q.t.p. em $[0, T], i \in\{1, \ldots, m\}$, tais que

$$
\begin{gathered}
\nabla \phi(\bar{z}(t), t)+\nabla h^{\prime}(\bar{z}(t), t) u(t)+\nabla g^{\prime}(\bar{z}(t), t) v(t)=0 \text { q.t.p. em }[0, T], \\
v_{j}(t) g_{j}(\bar{z}(t), t)=0 \text { q.t.p. em }[0, T], j \in\{1, \ldots, m\} .
\end{gathered}
$$

Além disso, existe $M>0$ tal que

$$
\|(u(t), v(t))\| \leq M K_{0} K_{\phi} \text { q.t.p. em }[0, T],
$$

$e$

$$
\int_{0}^{T} \gamma^{\prime}(t)\left\{\nabla^{2} \phi(z(t), t)+\nabla^{2} h^{\prime}(z(t), t) u(t)+\nabla^{2} g^{\prime}(\bar{z}(t), t) v(t)\right\} \gamma(t) d t \leq 0 \quad \forall \gamma \in \bar{N},
$$

onde $\bar{N}$ é dado por

$\bar{N}=\left\{\gamma \in L_{n}^{\infty}[0, T] \mid \nabla h(\bar{z}(t), t) \gamma(t)=0, \quad \nabla g_{j}^{\prime}(\bar{z}(t), t) \gamma(t)=0\right.$ q.t.p. em $\left.[0, T], j \in I_{a}(t)\right\}$.

Demonstração. Considera-se uma função mensurável $w:[0, T] \rightarrow \mathbb{R}^{m}, \bar{z}$ minimizador local para (PTC) e um problema auxiliar (AUX1) apenas com restrições de igualdade obtido de (PTC) por meio do uso de folgas complementares, e procede-se em vários passos.

Passo 1: Mostra-se que $(\bar{z}, \bar{w})$ é um minimizador local para (AUX1), onde $\bar{w}_{j}(t)=\sqrt{g_{j}(\bar{z}(t), t)}$ q.t.p. em $[0, T], j \in\{1, \ldots, m\}$.

Passo 2: Verifica-se que o problema auxiliar (AUX1) satisfaz as condições (H1)-(H4) para que o Teorema 3.2 seja aplicável.

Passo 3: Aplica-se o Teorema 3.2, obtendo os resultados desejados.

Passo 4: Usando a condição necessária de segunda ordem (2) do Teorema 3.2, verificase a não-negatividade dos multiplicadores das restrições de desigualdade e a condição necessária de segunda ordem para o problema (PTC). 


\section{Conclusões}

Condições necessárias de primeira e segunda ordens foram inicialmente obtidas para um problema de otimização não-linear com tempo contínuo irrestrito (SR). Com este resultado, a utilização de um Teorema da Função Implícita Uniforme [3] e uma qualificação de restrições tipo independência linear, condições necessárias de primeira e segunda ordens foram desenvolvidas para o problema com restrições de igualdade (RI).

Considerando o problema com restrições de igualdade e desigualdade (PTC) e utilizando folgas complementares nas restrições de desigualdade, o problema foi reformulado para o caso com restrições de igualdade (RI) e condições necessárias de primeira e segunda ordens foram obtidas. Nesse processo, novas ferramentas foram desenvolvidas para se atingir tais objetivos e ideias da programação não-linear em dimensão finita foram generalizadas para o caso tempo contínuo.

\section{Agradecimentos}

M. R. C. do Monte agradece a Deus, às orientações do prof. Dr. Valeriano Antunes de Oliveira, ao apoio da família, ao Ibilce-UNESP e a UFU.

V. A. de Oliveira foi parcialmente apoiado pelos processos de números 2013/07375-0 e 2016/03540-4 da Fundação de Amparo à Pesquisa do Estado de São Paulo (FAPESP), 457785/2014-4 e 310955/2015-7 do Conselho Nacional de Desenvolvimento Científico e Tecnológico (CNPq).

\section{Referências}

[1] R. Bellman, Bottleneck problems and dynamic programming, Proceedings of the National Academy of Sciences of the United States of America, 39:947-951, 1953.

[2] B. D. Craven and J. J. Koliha, Generalizations of Farkas Theorem, Siam J. Math. Anal, 8:983-997, 1977.

[3] M. R. De Pinho and R. B. Vinter, Necessary conditions for optimal control problems involving nonlinear differential algebraic equations, J. Math. Anal. Appl., 212:493$516,1997$.

[4] T. W. Reiland, Optimality Conditions and Duality in Continuous Programming I. Convex Programs and a Theorem of the Alternative. Journal of Mathematical Analysis an Applications, 77:297-325, 1980.

[5] W. Rudin, Principles of Mathematical Analysis, 3rd Edition, McGraw-Hill, New York, 1976.

[6] G. J. Zalmai, Sufficient optimality conditions in continuous-time nonlinear programming, Journal of mathematical analysis and applications, 111:130-147, 1985. 